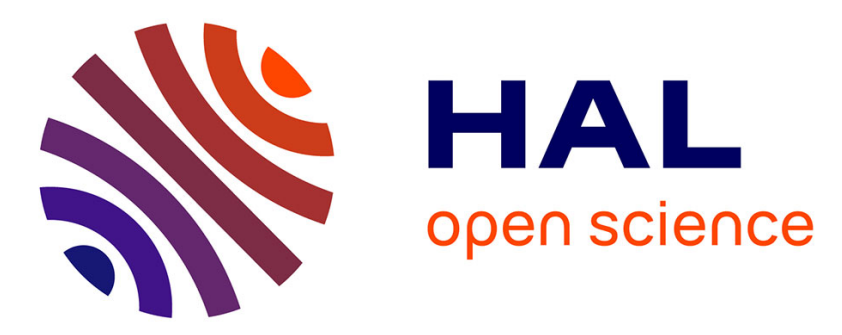

\title{
Muscle architecture and EMG activity changes during isotonic and isokinetic eccentric exercises
}

Gaël Guilhem, Christophe Cornu, Arnaud Guével

\section{To cite this version:}

Gaël Guilhem, Christophe Cornu, Arnaud Guével. Muscle architecture and EMG activity changes during isotonic and isokinetic eccentric exercises . European Journal of Applied Physiology, 2011, 111 (11), pp.2723-2733. 10.1007/s00421-011-1894-3 . hal-01566268

\section{HAL Id: hal-01566268 https: / hal-insep.archives-ouvertes.fr/hal-01566268}

Submitted on 20 Jul 2017

HAL is a multi-disciplinary open access archive for the deposit and dissemination of scientific research documents, whether they are published or not. The documents may come from teaching and research institutions in France or abroad, or from public or private research centers.
L'archive ouverte pluridisciplinaire HAL, est destinée au dépôt et à la diffusion de documents scientifiques de niveau recherche, publiés ou non, émanant des établissements d'enseignement et de recherche français ou étrangers, des laboratoires publics ou privés. 
Muscle architecture and EMG activity changesduring isotonic and isokinetic eccentric exercises

Gaël Guilhem, Christophe Cornu \& Arnaud Guével 



\title{
Muscle architecture and EMG activity changes during isotonic and isokinetic eccentric exercises
}

\author{
Gaël Guilhem · Christophe Cornu · Arnaud Guével
}

Abstract The present study aimed to compare muscle architecture and electromyographic activity during isotonic (IT) and isokinetic (IK) knee extensors eccentric contractions. Seventeen subjects were assigned in test and reproducibility groups. During test session, subjects performed two IT and two IK sets of eccentric contractions of knee extensor muscles. Torque, angular velocity, VL architecture and EMG activity of agonist (vastus lateralis, VL; vastus medialis; rectus femoris) and antagonist (semitendinosus; biceps femoris, BF) muscles were simultaneously recorded and averaged on a $5^{\circ}$ window. Torque-angle and angular velocity-angle relationships exhibited differences in mechanical load between the IT and IK modes. Changes in muscle architecture were similar in both modes, since VL fascicles length increased and fascicle angle decreased, resulting in a decrease in muscle thickness during eccentric contraction. Agonist activity and BF co-activity levels were higher in IT mode than in IK mode at short muscle lengths, whereas agonist activity was higher in IK mode than in IT mode at long muscle lengths. Differences in mechanical

Electronic supplementary material The online version of this article (doi:10.1007/s00421-011-1894-3) contains supplementary material, which is available to authorized users.

G. Guilhem · C. Cornu · A. Guével

Faculty of Sport Sciences, "Motricité, Interactions,

Performance" Laboratory, EA 4334,

University of Nantes, Nantes, France

G. Guilhem (\&)

Research Department,

National Institute for Sports (INSEP),

11, av du Tremblay, 75012 Paris, France

e-mail: gael.guilhem@insep.fr load between both modes induced specific neuromuscular responses in terms of agonist activity and antagonist coactivity. These results suggest that specific neural adaptations may occur after IT or IK eccentric training. This hypothesis needs to be tested in order to gain new insights concerning the most effective eccentric protocols based on whether the objective is sportive or clinical.

Keywords Ultrasonography - Electromyography ·

Coactivation $\cdot$ Lengthening contraction $\cdot$ Knee extensors

\section{Introduction}

Eccentric muscle contraction causes an active stretch of muscle fascicles during contraction (Enoka 1996). During this forced lengthening, elastic components of the muscletendon unit are stretched while the muscular fibers contract. These mechanical conditions due to eccentric contraction induce a higher torque force able to stimulate inhibition mechanism to protect contractile structures and tendons for high tension generated (Duchateau and Enoka 2008). For this reason this contraction is useful in training programs to increase muscle strength (Duclay et al. 2008; Housh et al. 1998) or in rehabilitation programs to restore muscle or tendon function (Visnes and Bahr 2007).

Isotonic (IT) eccentric programs are recognized as particularly effective in increasing maximal strength (Duclay et al. 2008; Housh et al. 1998). The development of isokinetic (IK) dynamometers allowed for application of an accommodating resistance to the muscles, resulting in eccentric exercise at a constant movement velocity. Comparative analyses of mechanical parameters during IT and IK eccentric exercises have shown different mechanical constraints in terms of external torque and angular 
movement velocity as a function of joint angles (Guilhem et al. 2010c). It is well known that external torque exerted by the knee extensors in the IT mode is constant throughout the entire range of motion, whereas it increases in the IK mode from short to long muscle length. Although each mode creates a different mechanical load on the muscles, to date, the specific effects of IT and IK eccentric exercises on neuromuscular and musculoskeletal systems have not been determined probably considering the difficulties to compare eccentric contraction in similar experimental conditions (Guilhem et al. 2010a). Yet, this comparison needs to standardize both modes to exclude non-controlled effects such as movement velocity, contraction duration or amount of mechanical work.

Numerous studies have focused on the behavior of muscle fascicles during eccentric contractions, using analysis of ultrasonographic images, in order to improve the understanding of the biomechanics behavior of muscle during locomotion. Some authors have shown that the lengthening of the muscle-tendon unit is completely supported by tendinous tissues (i.e., aponeurosis, tendon) during eccentric contraction of the plantar flexor muscles (Fukunaga et al. 2001). Consequently, the Achilles tendon behaves as a mechanical buffer. Recent studies have shown a parallel lengthening of the vastus lateralis and the patellar tendon in eccentric contraction (Ishikawa et al. 2003). These differences in findings could be due to the exercise mode, the exercise intensity and the muscles considered. Research studies conducted on the IK condition showed no effect of movement velocity on fascicle length, fascicle angle or muscle thickness during eccentric contractions (Reeves and Narici 2003). Recently, Pasquet et al. (2006) underscored the linear elongation of tibialis anterior fascicles during sub-maximal lengthening contractions performed at $10^{\circ} \mathrm{s}^{-1}$ (Pasquet et al. 2006). These results remain to be confirmed on maximal contractions and/or on other muscle groups.

The relationship between surface electromyographic signal (EMG) and joint angle showed a decrease of the EMG activity at short muscle length during IK eccentric contractions (Komi et al. 2000). Although EMG activity of the agonist muscles has been studied during IT eccentric contractions (Nakazawa et al. 1993; Nardone et al. 1989), to our knowledge no study has determined the muscle activation as a function of joint angle in this condition. A few mechanisms have been suggested as responsible for modulation of muscle activity during eccentric contraction: torque levels may be modulated by sensory $\mathrm{Ib}$ afferents from Golgi organs, group Ia and II muscle spindle afferents, group III muscle afferents and/or by recurrent inhibition (Duclay and Martin 2005).

The aim of the present study is to compare knee extensor muscles behavior during IT and IK standardized eccentric exercises. We hypothesize that differences in torque and angular velocity between the two standardized modes induce specific EMG patterns and muscle architecture changes of the knee extensor muscles as a function of muscle length.

\section{Methods}

Subjects

Seventeen healthy subjects with no previous history of knee injury volunteered to participate in this study. The participants were assigned either to a test group $(n=12)$ or to a reproducibility group $(n=5)$. The reproducibility group was constituted in order to test the reproducibility of architectural measurements in dynamic conditions. The mean $( \pm S D)$ age, height, body mass and body mass index (BMI) of the subjects were $22.6 \pm 2.8$ years, $179.3 \pm 6.0 \mathrm{~cm}, 76.6 \pm 8.9 \mathrm{~kg}$ and $23.8 \pm 2.2 \mathrm{~kg} \mathrm{~m}^{-2}$, respectively, for the test group, and $27.6 \pm 1.4$ years, $176.2 \pm 6.2 \mathrm{~cm}, 68.3 \pm 5.9 \mathrm{~kg}$ and $21.9 \pm 2.1 \mathrm{~kg} \mathrm{~m}^{-2}$ for the reproducibility group. All participants were informed regarding the nature, aims, risks and discomfort associated with the study before they gave their written consent to participate. The study was approved by the local ethics committee and was conducted in accordance with the Helsinki Declaration (1964, revised in 2001).

\section{Dynamometry}

Eccentric sessions were performed on a customized isokinetic dynamometer (Biodex ${ }^{\circledR}$ System 3 Pro, Shirley, NY, USA) that was previously validated (Guilhem et al. 2010b). In order to be able to compare the effect of IT and IK eccentric exercises under similar conditions, we recently proposed a procedure with the purpose of standardizing both exercise modes during eccentric contraction (Guilhem et al. 2010c). Briefly, this method consisted in equalizing the amount of work performed and the mean angular velocity in each mode, on the same ergometer. During exercise, subjects were seated on the ergometer so that the hip was flexed to $85^{\circ}\left(0^{\circ}=\right.$ full hip extension $)$ and the range of motion was fixed from $30^{\circ}$ to $90^{\circ}$ knee angle $\left(0^{\circ}=\right.$ full leg extension $)$. In the IT mode, subjects performed eccentric contractions of the knee extensors against a constant torque applied by a plate-loaded resistance training device throughout the entire range of motion. In the IK mode, subjects performed eccentric contractions at a constant angular velocity. This approach allowed us to stop the exercise when a preset amount of angular work was achieved. Ergometer settings were recorded and reproduced for all sessions. Mechanical signals were recorded at 
a sampling frequency of $1,000 \mathrm{~Hz}$. Torque signals were gravity and inertia corrected through the overall range of motion (Aagaard et al. 1995; Guilhem et al. 2010b). The amount of work $(\mathrm{W})$ and the mean angular velocity $\partial_{X} P$ were determined for each repetition. In the IT and IK modes, torque and angular velocity were averaged every $5^{\circ}$ between $30^{\circ}$ and $90^{\circ}$ on a $5^{\circ}$ window (i.e., $2.5^{\circ}$ before and $2.5^{\circ}$ after each target angle). Finally, the values of the two sets were averaged for each repetition in both modes.

\section{Experimental design}

Each subject of the test group participated in a familiarization session and a test session 1 week apart.

\section{Familiarization session}

The familiarization session allowed the subjects to become accustomed to the customized ergometer and the IT and IK modes. After $5 \mathrm{~min}$ of warm-up on a cycloergometer (100 Watts) and a specific warm-up on the ergometer, subjects executed three maximal concentric flexions and three maximal IK eccentric extensions at $30^{\circ} \mathrm{s}^{-1}$ to determine the joint angle corresponding to peak torque for knee extensors and knee flexors. Then 1RM was determined as the last load the subjects could lift once on the whole range of motion with a $5^{\circ}$ tolerance. Then subjects completed eight eccentric contractions of the knee extensor muscles in the IT mode at $120 \%$ of $1 \mathrm{RM}$. After 5 min of rest, they completed $n$ maximal eccentric contractions of the knee extensor muscles in the IK mode, from $30^{\circ}$ to $90^{\circ}$ knee angle at the same mean angular velocity that was calculated in the IT set. In both modes, subjects had to contract their knee extensors maximally and the movement was triggered as soon as they produced muscle force. In these conditions, both mechanical work and duration of contraction were the same in each exercise mode. $n$ was the number of repetitions performed in the IK mode so the set was stopped when the same amount of work performed in the IT set was achieved (Guilhem et al. 2010c).

\section{Test session}

Test session was dedicated to the investigation of muscle architecture and muscle activity changes during IT and IK eccentric contractions. Subjects warmed up and the 1RM was determined as described above. The maximum voluntary isometric torque was assessed for the knee extension $\left(\mathrm{MVC}_{\mathrm{Ext}}\right)$ and knee flexion $\left(\mathrm{MVC}_{\mathrm{Flex}}\right)$ at the individual angle at which the peak torque was obtained during the familiarization session. Three MVC trials of $5 \mathrm{~s}$ were completed for each angle, with $30 \mathrm{~s}$ of rest between trials and 2 min of rest between $\mathrm{MVC}_{\mathrm{Ext}}$ and $\mathrm{MVC}_{\mathrm{Flex}}$. Then subjects performed one IT set at $120 \%$ of $1 \mathrm{RM}$ and one IK set of $n$ maximal eccentric contractions of the knee extensor muscles with $5 \mathrm{~min}$ of rest between the two sets. After $5 \mathrm{~min}$ of rest, subjects performed exactly the same sequence of one IT set and one IK set, thus performing a total of 2 IT sets and 2 IK sets.

Images acquisition

The muscle architecture changes of the VL during eccentric contractions were examined in vivo by ultrasonography (Blazevich et al. 2006; Ishikawa et al. 2003; Kubo et al. 2003). Longitudinal images were obtained using real-time a two-dimensional (2-D) B-mode ultrasonographic apparatus (Philips HD3, Bothell, WA, USA) with a $40 \mathrm{~mm}$ linear-array probe $(7.5 \mathrm{MHz})$. The probe position was directed onto the right leg at $50 \%$ of the distance from the central palpable point of the greater trochanter to the lateral condyle of the femur at a $90^{\circ}$ knee angle (Blazevich et al. 2006; Fukunaga et al. 1997; Ichinose et al. 2000). The echoes reflected from the superficial and deep aponeuroses and the interspaces among the fascicles clearly defined muscle thickness $(t)$, fascicle angle (h) and fascicle length $\left(L_{\mathrm{f}}\right)$. Once muscle fascicles were clearly identified on the echographic images, the probe was firmly fixed in position using a custom-made plastic sheath strapped around the thigh. The restraint ensured a constant orientation (perpendicular to the skin) of the probe. The internal surface of the plastic sheath was covered by a thin foam layer fixed onto the skin by a double-faced adhesive tape in order to avoid any movement of the probe during the recordings. The probe and plastic sheath positions were labeled at the start of the test session to ensure that the probe was in the same position at the end of the measurements. A watersoluble gel was applied to the transducer to provide acoustic coupling and so there was no need to contact the skin. Images were acquired at a sampling rate of $25 \mathrm{~Hz}$ with commercialized software (Pinnacle Studio, v 10.0).

\section{Architectural measurements}

For the test group, in the IT mode the repetition performed at an angular velocity closest to the mean angular velocity of the first set was selected for further analysis. In the IK mode, the repetition with the amount of work closest to the mean amount of work performed in one repetition in the first set was selected. Images corresponding to each target angle (every $5^{\circ}$ ) of the selected repetitions were extracted from ultrasonographic videos. Muscle thickness, fascicle angle and fascicle length were measured from each VL ultrasound scan (Blazevich et al. 2006; Fukunaga et al. 1997; Ichinose et al. 2000). A muscle fascicle was defined 
as a clearly visible fiber bundle lying between the superficial and the deep aponeuroses. For each scan, three muscle fascicles were clearly labeled using drawing software (Photoshop Elements v 4.0, Adobe System Inc., San Jose, CA, USA). The aponeuroses and muscle fascicles were then digitized using a public domain image-processing software (Image J, National Institute of Health, Bethesda, MD, USA) (Fig. 1). Measurements of $t$, defined as the distance between the superficial and the deep aponeuroses, were made on both sides of the image and averaged (Reeves and Narici 2003). The fascicle angle was determined as the angle between the fascicle and its insertion on the deep aponeurosis (Blazevich et al. 2006; Fukunaga et al. 1997). $L_{\mathrm{f}}$ was defined as the length of the fascicular path between the superficial and the deep aponeurosis. Because VL fascicles were too long to be captured fully by our probe and so were impossible to measure from origin to insertion, its length $\left(L_{\mathrm{f} 1}\right)$ was estimated using trigonometry according to the following Eq. 1, assuming a linear continuation of the fascicles (Blazevich et al. 2006; Reeves and Narici 2003):

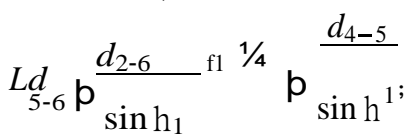

where $d_{5-6}$ represents the visible part of the fascicule, and $d_{4-5}$ and $d_{2-6}$ represent the distance between the visible

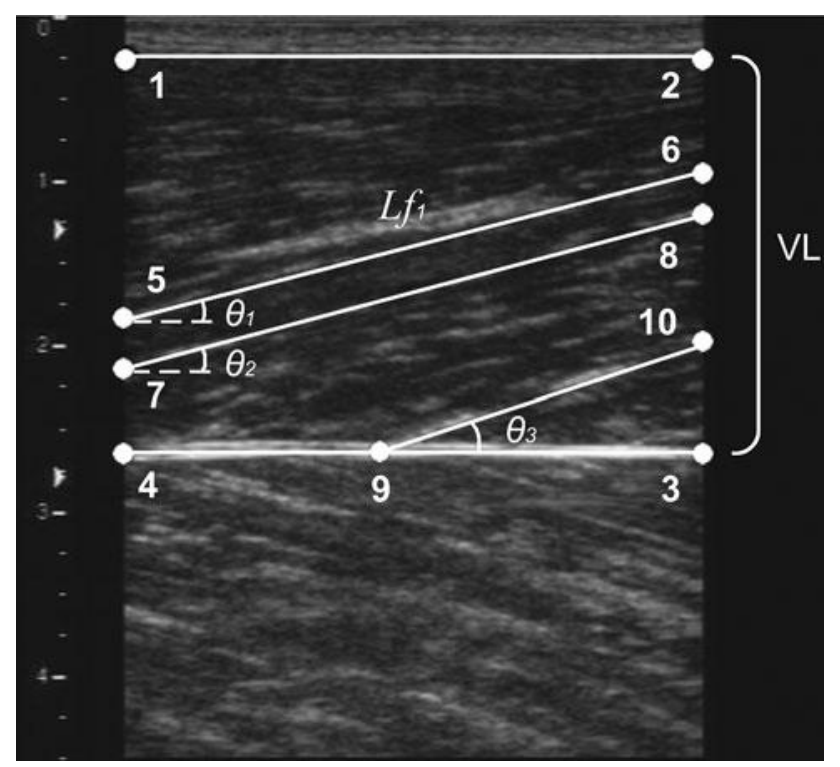

Fig. 1 Sagittal plane ultrasounds scan of vastus lateralis (VL). Landmarks corresponding to aponeuroses (points 1-4) and fascicles (points 5-10) were digitized (see "Methods"), as in the image. Muscle thickness was calculated as the mean of the distances 1-4 $\left(d_{1-4}\right)$ and 2-3 $\left(d_{2-3}\right)$. Fascicle length was determined according to the

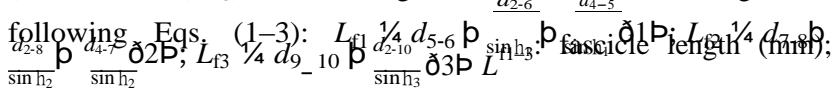
$\mathrm{h}_{1-3}$ : fascicle angle (rad); $d_{5-6}$ : distance between points 5 and $6(\mathrm{~mm})$ extremity of the fascicule and the aponeurosis (see Fig. 1 for the complete calculations).

The error that is due to the linear extrapolation has been estimated at $2-7 \%$ with similar methods of estimating gastrocnemius medialis (MG) fascicles length when the entire fascicle cannot be visualized (Abellaneda et al. 2009). Whether MG fascicles are reported to be shorter than VL fascicles (i.e., $60 \mathrm{~mm}$ for $\mathrm{MG}$ fascicles vs. an average of $105 \mathrm{~mm}$ for VL fascicles in our study), similar ultrasound probe field of view has previously been used to measure VL fascicles with reproducible results (Blazevich et al. 2006). In order to limit the effect of estimation, the values of three measured fascicles for each repetition were averaged to obtain a representative value of $L_{\mathrm{f}}$ for each scan. For the reproducibility group all repetitions were considered for further analysis. Images corresponding to the target angles were extracted from each repetition, labeled and digitized as described for the test group. The five values of $t, \mathrm{~h}$ and $L_{\mathrm{f}}$ were averaged for each angle.

Reproducibility of architectural measurements

The subjects assigned to the reproducibility group participated in two identical sessions with 1 day in between in order to determine the reproducibility of the architectural measurements (i.e., $t, \mathrm{~h}$ and $L_{\mathrm{f}}$ ) during eccentric contractions. In both sessions, subjects warmed up and the 1RM was determined as described for the test group. Then they performed four eccentric sets: one IT set of five repetitions at $120 \%$ of $1 \mathrm{RM}$ and three IK sets of five repetitions each at 10,30 and $60^{\circ} \mathrm{s}^{-1}$. These angular velocities were chosen to cover the lower, the higher and the mean velocities recorded during test session. All sets were performed in a randomized order, and the set order used in the first session was repeated in the second session for each subject.

\section{Electromyography}

Bipolar surface electromyography (EMG) signals were recorded from surface EMG sensors (Delsys, Boston, MA, USA) during a test session on the vastus lateralis (VL), vastus medialis (VM), rectus femoris (RF), semitendinosus (ST) and long head biceps femoris (BF) muscles. Each EMG sensor was made of two parallel silver bars with a length of $10 \mathrm{~mm}$. The inter-bar distance was $10 \mathrm{~mm}$. Electrode-skin impedance was reduced using standard skin preparation procedures (Hermens et al. 2000). According to the SENIAM recommendations (Hermens et al. 2000), surface EMG sensors were placed between the distal tendon and the innervation zone parallel to the direction of the

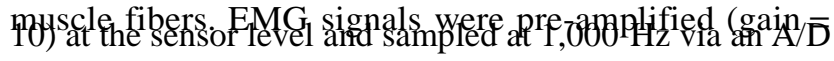
converter (Bagnoli 16 EMG System, Delsys, Boston, MA, 
USA; gain $=1,000 ;$ bandwidth $=6-450 \mathrm{~Hz}$ ). In order to synchronize mechanical and electromyographic data with ultrasonographic videos, the signal of the switch used to start the video was also recorded using the Delsys system. The video was started by pressing a pedal which switch off a signal on the video and this moment was also recorded by the Delsys system on a trigger channel.

\section{EMG processing}

$\mathrm{MVC}_{\text {Ext }}$ and $\mathrm{MVC}_{\mathrm{Flex}}$ trials with the highest maximal torque were considered for further analysis. The maximal root mean square (RMS) value was calculated over a time period of $200 \mathrm{~ms}$ (i.e., $100 \mathrm{~ms}$ before and after the time to peak torque) from EMG signals of each knee extensor muscle (i.e., VL, VM and RF) for $\mathrm{MVC}_{\text {Ext }}$ and each knee flexor muscle (i.e., ST and $\mathrm{BF}$ ) for $\mathrm{MVC}_{\mathrm{Flex}}$ (Remaud et al. 2009). For each IT and IK eccentric contraction, a mean RMS value was first calculated on the range of motion comprising isotonic and isokinetic steady states (from a $35^{\circ}$ to an $80^{\circ}$ knee angle). Second, an RMS value was calculated for each muscle every $5^{\circ}$ and normalized to the maximal RMS value obtained during corresponding MVC, and expressed as a percentage. Finally, the values of the two sets were averaged for each repetition in both modes.

\section{Statistical analysis}

Normality of data was tested using a Kolmogorov-Smirnov test. The level of concordance for the amount of work and the mean angular velocity between the IT and IK modes was assessed using Bland-Altman plots (Bland and Altman 1986). Two-way ANOVAs (mode 9 angle) for repeated measures were used to test differences in torque, angular velocity, duration of contraction, muscle thickness, fascicle angle, fascicle length, and EMG activity in agonist muscles and antagonist muscles between the two modes as a function of joint angle. Two separate three-way ANOVAs (mode 9 muscle 9 angle) for repeated measures were performed to test the effect of mode, muscle and knee joint angle on agonist muscle activity (RMS values from VL, $\mathrm{VM}$ and RF) and on antagonist muscle co-activity levels (RMS values from ST and BF). When the sphericity assumption in repeated ANOVAs was violated (Mauchly's test), then a Geisser-Greenhouse correction was used. Post hoc tests were performed by means of Newman-Keuls procedures. Linear regressions between the EMG activity and torque, and between EMG activity and angular velocity values were performed and coefficients of determination $\left(R^{2}\right)$ were calculated. In order to have a reproducibility of the architectural parameters, ICC (interclass correlation coefficient), standard error of measurement (SEM) and the coefficient of variation (CV) were determined (Hopkins 2000). For all tests, the significance level was set at $P W 0.05$. All results are presented as mean $\pm \mathrm{SE}$.

\section{Results}

\section{Standardization procedure}

The Bland-Altman analysis revealed concordance between the IT and IK modes for the amount of work and for the mean angular velocity, as shown by the low bias values for the amount of work $(6.5 \mathrm{~J} ; 0.3 \%)$ and angular velocity $\left(-0.2^{\circ} \mathrm{s}^{-1} ; 0.5 \%\right)$ (Electronic Supplementary Material). The duration of contraction was not statistically different between IT and IK repetitions as revealed by variance analysis $(P=0.67)$. Mean amounts of work performed by a set during the IT and IK tests were -1,890.2 \pm 336.9 and $-1,896.7 \pm 334.1 \mathrm{~J}$, respectively. In these standardized conditions, no statistical differences were observed in the number of repetitions performed to achieve the same amount of work in the IT and IK modes (8.0 vs. $8.0 \pm 0.6$, respectively; $P=0.92$ ).

Torque-angle and angular velocity-angle relationships

The two-way ANOVAs revealed a mode 9 angle interaction effect for the knee extensors' torque-angle $(P W 0.0001)$ and velocity-angle $(P W 0.0001)$ relationships. The torques developed during eccentric contractions in the IT mode were higher than those produced in the IK mode from $30^{\circ}(? 393 \%)$ to $50^{\circ}$ (?10.9\%), while torques were higher in the IK mode than in the IT mode from $60^{\circ}$ $(? 12.6 \%)$ to $85^{\circ}(? 28.8 \%)$, as revealed by the post hoc analysis (Fig. 2a). The analysis of the velocity-angle relationship showed that angular velocity averaged on $5^{\circ}$ windows was higher in the IT mode than in the IK mode from $30^{\circ}$ to $60^{\circ}$ (Fig. 2b).

\section{Muscle architecture changes}

Reproducibility values of muscle architecture parameters are presented in Table 1. ICCs were very high for muscle thickness (from 0.93 to 0.95 ) and high for the fascicle angle (from 0.75 to 0.87 ) and fascicle length (from 0.82 to 0.88 ). The standard error between repeated measurements for muscle thickness, fascicle angle and fascicle length ranged from 1.3 to $1.4 \mathrm{~mm}, 1.1^{\circ}$ to $1.4^{\circ}$ and 9.2 to $11.9 \mathrm{~mm}$, respectively (Table 1) (Sleivert and Wenger 1994). Changes in muscle thickness, fascicle angle and fascicle length changes are presented in Fig. 3. Eccentric contractions in the IT and IK modes induced similar muscle architecture behavior since the two-way ANOVAs did not show any 

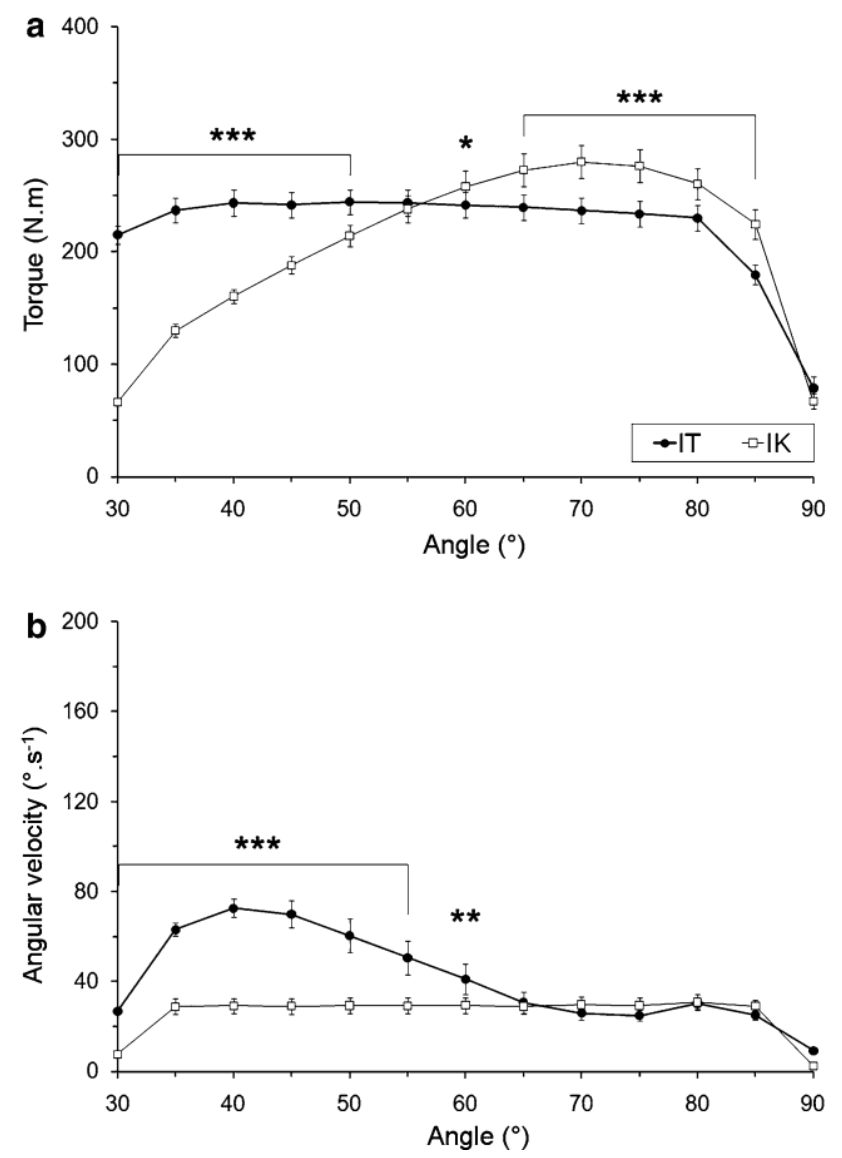

Fig. 2 Torque-angle (a) and angular velocity-angle (b) relationships during eccentric contraction of the knee extensor muscles performed in isotonic (IT, filled circle) and isokinetic (IK, unfilled square) modes (sets are pooled, $0^{\circ}=$ full leg extension). ${ }^{*}$ Significant differences between IT and IK modes (*PW 0.05 ; **PW 0.01 ; ***PW0.001)

effect of the mode on muscle thickness $(P=0.33)$, fascicle angle $(P=0.51)$ or fascicle length $(P=0.55)$ as a function of knee joint angle (Fig. 3). During eccentric contractions (the IT and IK modes pooled), muscle thickness decreased from $60^{\circ}(-3.2 \pm 0.9 \%)$ to $90^{\circ}(-4.2 \pm 1.2 \%$; $P W 0.05$; Fig. 3a), fascicle angle decreased from $60^{\circ}$ $(-12.9 \pm 2.3 \%)$ to $90^{\circ} \quad(-30.0 \pm 5.4 \% ; \quad P W 0.05$; Fig. 3b), and fascicle length increased from $70^{\circ}$ (?19.8 \pm $4.5 \%)$ to $90^{\circ}(? 47.4 \pm 10.7 \%)$ in comparison to the $30^{\circ}$ knee angle ( $P W 0.05$; Fig. 3c).

\section{Agonist EMG activity changes}

Linear regressions showed a significant correlations $(P W 0.001)$ between torque and EMG activity levels ( $R^{2}=0.94$ for IT mode; $R^{2}=0.91$ for IK mode) and between angular velocity and EMG activity levels $\left(R^{2}=0.88\right.$ for IT mode; $R^{2}=0.91$ for IK mode) for both modes on the whole range of motion. IT eccentric exercises elicit higher mean EMG activity of the quadriceps femoris
Table 1 Reliability of vastus lateralis architecture parameters

\begin{tabular}{llllc}
\hline Parameter & Exercise mode & ICC & SEM & CV (\%) \\
\hline Muscle thickness $(t)$ & IT $120 \%$ 1RM & 0.94 & $1.3 \mathrm{~mm}$ & 5.4 \\
& IK $10^{\circ} \mathrm{s}^{-1}$ & 0.95 & $1.2 \mathrm{~mm}$ & 5.3 \\
& IK $30^{\circ} \mathrm{s}^{-1}$ & 0.93 & $1.4 \mathrm{~mm}$ & 6.1 \\
& IK $60^{\circ} \mathrm{s}^{-1}$ & 0.94 & $1.3 \mathrm{~mm}$ & 5.2 \\
Pennation angle (h) & IT $120 \% 1 \mathrm{RM}$ & 0.85 & $1.2^{\circ}$ & 9.6 \\
& IK $10^{\circ} \mathrm{s}^{-1}$ & 0.87 & $1.1^{\circ}$ & 9.3 \\
& IK $30^{\circ} \mathrm{s}^{-1}$ & 0.75 & $1.4^{\circ}$ & 12.4 \\
& IK $60^{\circ} \mathrm{s}^{-1}$ & 0.83 & $1.3^{\circ}$ & 10.4 \\
Fascicle length $\left(L_{\mathrm{f}}\right)$ & IT $120 \% 1 \mathrm{RM}$ & 0.88 & $9.2 \mathrm{~mm}$ & 8.3 \\
& IK $10^{\circ} \mathrm{s}^{-1}$ & 0.87 & $9.5 \mathrm{~mm}$ & 8.6 \\
& IK $30^{\circ} \mathrm{s}^{-1}$ & 0.82 & $11.9 \mathrm{~mm}$ & 11.3 \\
& IK $60^{\circ} \mathrm{s}^{-1}$ & 0.85 & $10.3 \mathrm{~mm}$ & 10.1 \\
\hline
\end{tabular}

Intraclass correlation coefficient (ICC), standard error of measurement (SEM) and coefficient of variation (CV) of muscle thickness, pennation angle and fascicle length measured during eccentric contractions in isotonic (IT) mode at $120 \%$ of the maximal repetition (1RM) and isokinetic (IK) mode at 10,30 and $60^{\circ} \mathrm{s}^{-1}$, with 1 day between measurement session

muscles than IK eccentric exercise does $(78.7 \%$ in IT mode vs. $75.2 \%$ in IK mode; $P W 0.001)$ without a muscle 9 mode interaction $(P=0.28)$ (Fig. $4 \mathrm{a})$. The results also indicated an angle-specific response of the agonist EMG activity. The two-way ANOVA for myoelectrical activity of knee extensor muscles revealed a significant mode 9 angle effect $(P W 0.0001)$ and VL (Figs. 3d, 5a), VM and RF muscles behaved similarly (no muscle effect, $P=0.90$ ) (Fig. 5b, c). The normalized RMS value of agonist muscles was, on average, $26.9 \%$ higher in the IT mode than in the IK mode from the $30^{\circ}$ knee angle $(? 51.9 \pm 16.2 \%)$ to the $50^{\circ}$ knee angle $(? 10.3 \pm 7.0 \%)$ during eccentric contractions, as shown in Fig. 5a, b and c ( $P W 0.001)$. Agonist EMG activity was, on average, $18.8 \%$ higher in the IK mode than in the IT mode from the $70^{\circ}$ knee angle $(? 12.0 \pm 16.4 \%)$ to the $85^{\circ}$ knee angle $(? 33.1 \pm 17.6 \%)$ (PW0.001).

Antagonist EMG activity changes

Mean co-activity levels of the hamstring muscles were higher in the IT mode than in the IK mode ( $8.3 \%$ in the IT mode vs. $7.8 \%$ in the IK mode; $P W 0.001$ ) (Fig. $4 \mathrm{~b}$ ). The two-way ANOVA for EMG activity of the antagonist muscles (ST and BF) revealed a mode 9 muscle 9 angle effect $(P=0.01)$. Muscle activity of ST was not statistically different between the IT and IK modes during eccentric contractions (Fig. 5d). The RMS activity of the BF muscle was significantly higher in the IT mode from the $30^{\circ}$ knee angle $(? 30.2 \pm 13.0 \%)$ to the $55^{\circ}$ knee angle (?15.4 $\pm 8.3 \%$ ) (Fig. 5e). 

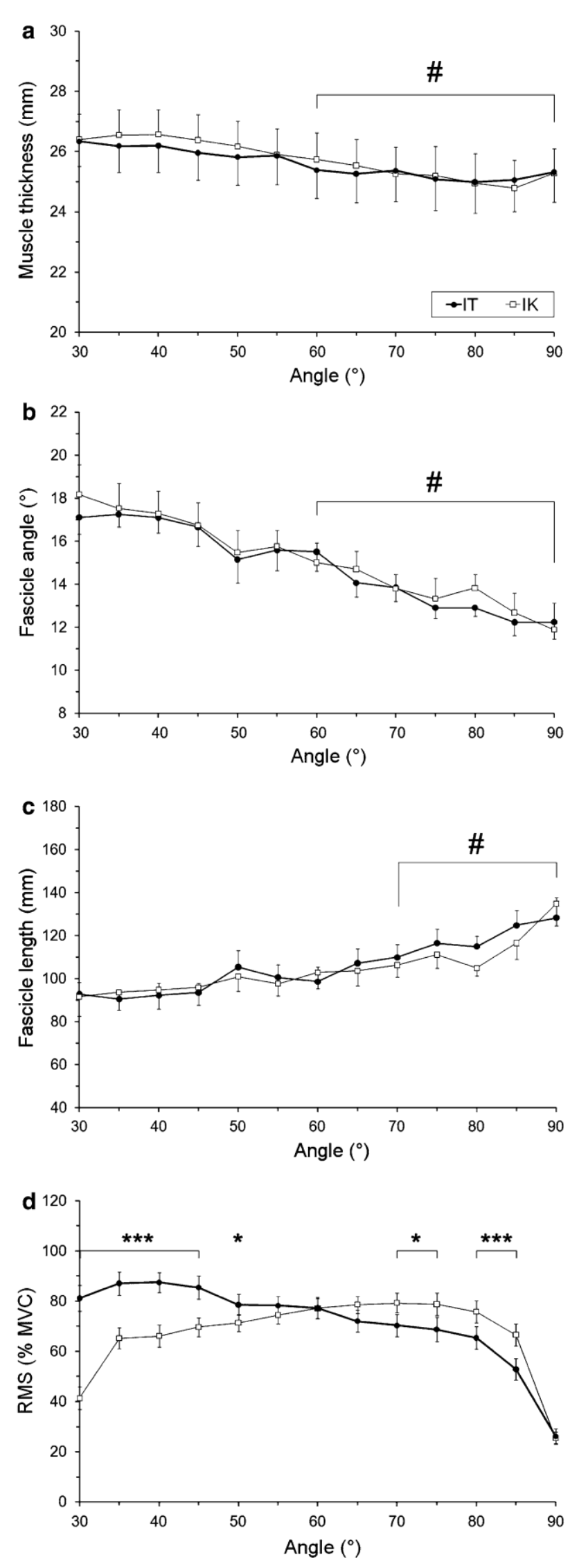

b Fig. 3 Changes in muscle thickness (a), fascicle angle (b), fascicle length (c) and root mean square (RMS) of the electromyographic activity (d) of the vastus lateralis during eccentric contraction of the knee extensor muscles performed in isotonic (IT, filled circle) and isokinetic (IK, unfilled square) modes (sets are pooled) from $30^{\circ}$ to $90^{\circ}$ knee joint angle $\left(0^{\circ}=\right.$ full leg extension). RMS value was normalized to the maximal RMS value obtained during the corresponding maximal isometric contraction (MVC). *Significant differences between modes (*P $P 0.05 ; * * * P W 0.001)$; ${ }^{*}$ significantly different from $30^{\circ}$ value $(P W 0.05)$

\section{Discussion}

The present study compared the behavior of knee extensor muscles during IT and IK eccentric exercises performed in standardized conditions in terms of the amount of work and the mean angular velocity. Our results show that the differences in mechanical load (i.e., instantaneous torque and angular velocity) induced by each exercise mode (Guilhem et al. 2010c) are associated with a specific pattern of the neural drive, as illustrated by different EMG activity and co-activity levels as a function of joint angle. Muscle architecture exhibited similar changes in IT and IK eccentric exercises.

EMG activity of the quadriceps femoris muscles was higher in the IT than in the IK mode during eccentric contraction. This result, which confirms previous observations obtained during concentric contraction (Remaud et al. 2009), can be attributed to the significant increase in angular velocity that occurs during an isotonic movement over that in an isokinetic movement (Fig. 2), since angular velocity has been shown to influence the agonist muscles' EMG activity (Babault et al. 2003). The agonist muscles' EMG activity level was the greatest in the IT mode at short muscle lengths and the greatest in the IK mode at long muscle lengths. The difference in EMG activity at the onset of the lengthening contraction could partly be due to increased motor unit discharge rate caused by the faster muscle stretch in IT mode (Pasquet et al. 2006). Moreover, the nature of external force may have different effect on the neural control of movement. Indeed, IT mode induces a loaded-contraction that involves a sufficient pre-activation level to control the movement (Junior et al. 2010), whereas the onset of isokinetic movement is unloaded until muscles start to produce external torque which triggers the movement. Both modes induced a decrease in EMG activity after the peak torque angle $\left(70^{\circ}\right)$. The mechanism responsible for causing a reduction in muscle activation when the muscle is stretched remains unclear, although some suggestions have been made in the literature (Duchateau and Enoka 2008). The type Ib afferents from the Golgi tendon organ (sensitive to muscle tension) can have an inhibitor impact on the motoneurons activity. However, the impact from the Golgi tendon organ does not seem to be the 

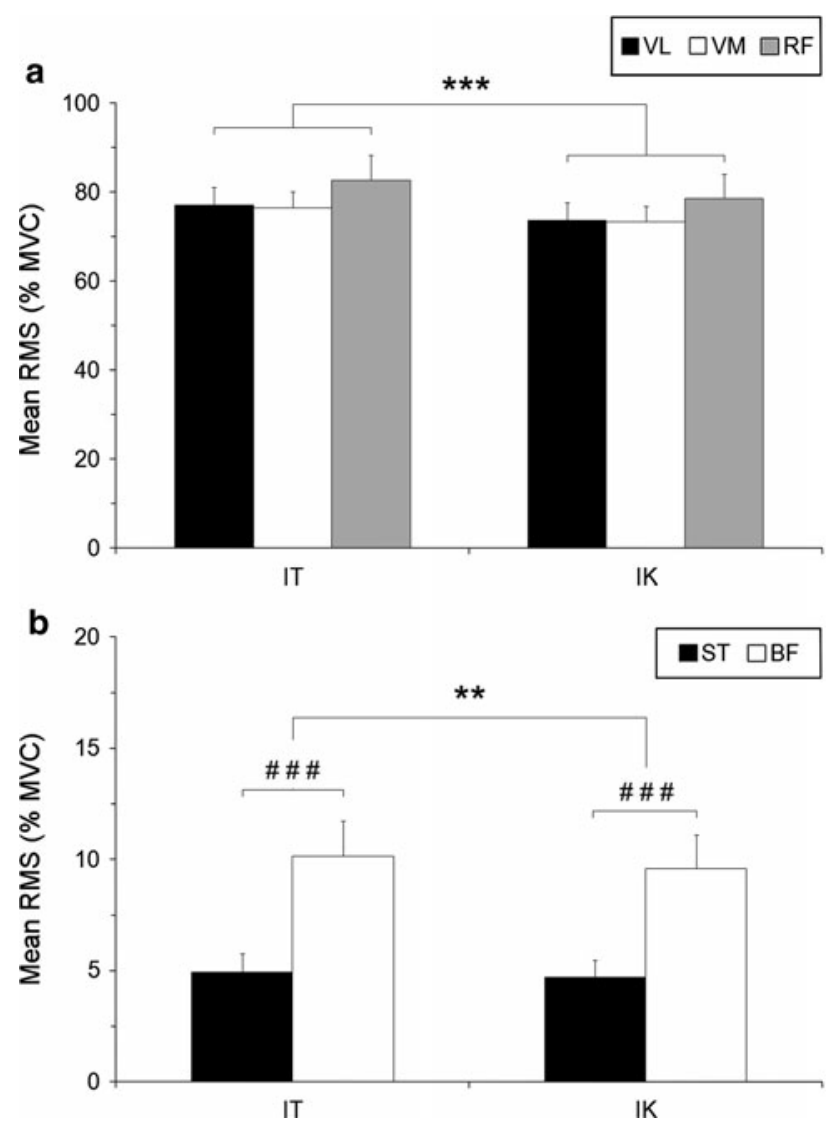

Fig. 4 Mean root mean square (RMS) value calculated from the surface electromyographic signals of agonist (a vastus lateralis, VL; vastus medialis, VM; rectus femoris, $R F$ ) and antagonist muscles (b semitendinosus, $S T$; biceps femoris, $B F$ ) during eccentric contraction of the knee extensor muscles performed in isotonic (IT) and isokinetic $(I K)$ modes (sets and repetitions are pooled). RMS value was normalized to the maximal RMS value obtained during the corresponding maximal isometric contraction (MVC). *Significant differences between modes $\left(* * P_{\#} W 0.01 ; * * * P W 0.001\right)$; ${ }^{*}$ significant differences between muscles ( $\left.{ }^{\#} P W 0.001\right)$

primary mechanism that can explain this activation decrease during an eccentric contraction (Pinniger et al. 2000). The efferent command can also be modulated by the Ia and II afferents stemming from the neuromuscular spindles that give information to the nervous system on muscle length (static sensitivity) and length variation (dynamic sensitivity). Previous investigations have reported that the pattern of motor-unit activity is related to muscle length (Babault et al. 2003; Pasquet et al. 2005). To our knowledge, the current study is the first to investigate the relationship between EMG activity and angle during IT eccentric contractions. We observed that the differences in mechanical load between the IT and the IK modes share specific patterns of the neuromuscular system, with higher agonist activity levels in the IT mode at short muscle lengths and higher activity levels in the IK mode at long muscle lengths, which is in agreement with the EMG- torque relationship described in the literature (Bigland and Lippold 1954). Previous works on elbow flexor muscles have observed a reduction in the amplitude of the average EMG during a single maximal IK eccentric contraction at long muscle lengths (Komi et al. 2000). These authors investigated a different muscle (i.e., biceps brachii) and followed a different protocol than is addressed in the current study (i.e., one maximal eccentric contraction, mean value on several repetitions, no visual feedback in real time on the exerted muscular force), which may partly explain the differences in their findings compared with our study. Such differences may also illustrate the differences in neuromuscular strategies used to overcome the external load in each mode. A higher activation level seems to be necessary in the IT mode to control the constant external load at the start of the angular course, whereas in the IK mode the accommodating resistance induces a lower activity level and, consequently, produces lower torque levels.

Differences in agonist EMG patterns are concomitant to superior co-activity levels in the IT mode in comparison to the IK eccentric exercise. The higher quadriceps activity in the IT mode compared to the IK mode could lead to a change in hamstring muscles' co-activity because of the "common drive" generated at the supra-spinal level, as many authors have proposed (Mullany et al. 2002). It would control the recruitment of both agonist and antagonist motoneurons pools. Moreover, BF activity was higher at short muscle length in the IT mode than in the IK mode during eccentric contractions (Fig. 5e). The high torque levels and the higher agonist EMG activity reached in the IT mode in comparison to the IK mode at these specific knee angles may induce a higher co-activity of this knee flexor muscle. This neural drive may be used to protect the joint and muscle structures against excessively the high tension levels that can occur during IT eccentric actions (Kellis and Baltzopoulos 1998). The lower co-activity levels found in our study (6.7 and 5.8\%) in comparison to other investigations (Guilhem et al. 2010a) may originate from the normalization method since co-activity levels were normalized by the activity recorded during maximal isometric flexion. It appears that this normalization procedure method is appropriate for normalizing the amplitude of EMG in order to compare muscles, tasks and individuals, or to retain the natural variations among individuals, as in our study (Burden and Bartlett 1999).

Muscle architecture has been investigated by the same method previously used on plantar flexor (Reeves and Narici 2003) and knee extensor muscles (Blazevich et al. 2006). The results obtained in dynamic conditions were also at least comparable to the results of previous studies performed in static conditions (Blazevich et al. 2006), regardless of the angular velocity tested. If the error due to 

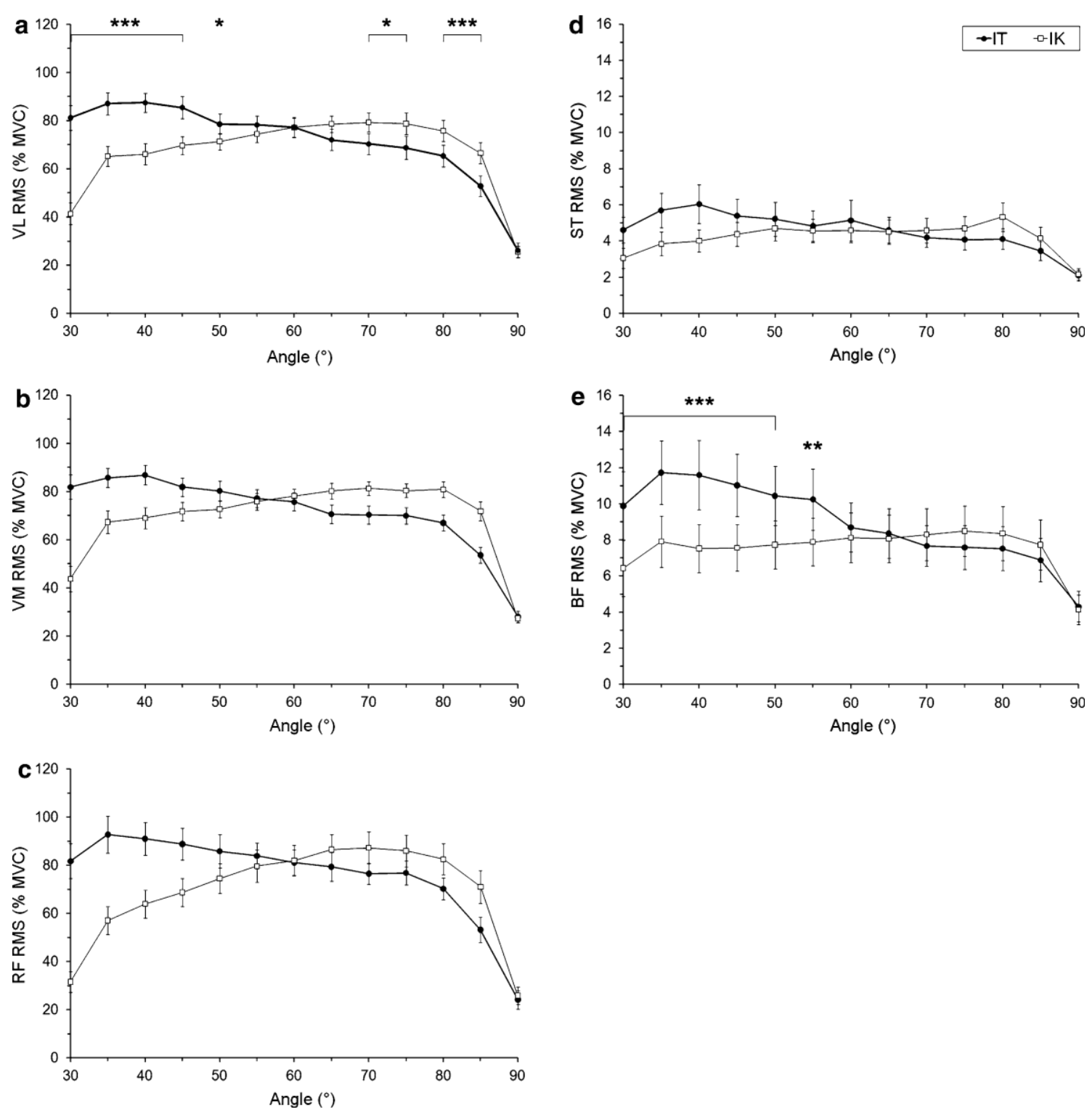

Fig. 5 Changes in root mean square (RMS) value calculated from the surface electromyographic signals of agonist (a vastus lateralis, $V L$; b vastus medialis, VM; c rectus femoris, $R F$ ) and antagonist (d semitendinosus, $S T$; e biceps femoris, $B F$ ) muscles during eccentric contraction of the knee extensors performed in isotonic (IT, filled

the linear extrapolation could have an effect on fascicle length measurements, we could assume that this effect is constant between IT and IK modes, as illustrated by the reproducibility results which are similar to previous investigations (Blazevich et al. 2006). This effect has been limited by measuring three fascicles per image and the probe was placed in a medial position, in order to ensure that the observed changes in fascicle angle with joint angle were not the result of a rotation of the muscle relative to the probe (Herbert and Gandevia 1995).

The values of VL fascicle length obtained in our study (from $93 \mathrm{~mm}$ at short muscle length to $128 \mathrm{~mm}$ at long

circle) and isokinetic (IK, filled square) modes (sets and repetitions are pooled, $0^{\circ}=$ full leg extension). RMS value was normalized to the maximal RMS value obtained during the corresponding maximal isometric contraction (MVC). *Significant differences between modes (*PW 0.05; **PW 0.01; ***PW0.001)

muscle length angles in the IT eccentric mode vs. 92$135 \mathrm{~mm}$ in the IK eccentric mode) are in accordance with previous studies that have determined the fascicle length of $\mathrm{VL}$ on the same range of motion (i.e., from $92 \mathrm{~mm}$ at $30^{\circ}$ to $117 \mathrm{~mm}$ at $90^{\circ}$ ) during isometric submaximal contractions (Fukunaga et al. 1997). Our results show an increase of fascicle length during eccentric contractions (from short to long muscle length), in both the IT and the IK mode. Given the fixed knee and hip angles during IT and IK exercise, it could be expected that differences in muscle force would induce differences in fascicles length due to strain of the elastic tissues in series with the muscle 
fascicles. Our results obtained on VL fascicles did not confirm this hypothesis, probably because of the muscle function of other agonist muscles (i.e., VM, RF, vastus intermedius) acting to the knee extension, which are not investigated in our study for methodological reasons. Additional evidence is that preload may have stretched the slack elements in IT mode and therefore allows greater force transmission at the beginning of the movement (Junior et al. 2010). Such mechanism may explain the absence of a difference in muscle architecture changes for the two contraction modalities. Reeves and Narici (2003) showed that TA fascicles linearly lengthen in submaximal lengthening contraction when this parameter is considered as a function of joint angle. These results are in accordance with our study, which shows similar behavior for the VL fascicles of the knee extensors, thereby confirming an active elongation of muscle fascicles during submaximal and maximal eccentric contraction.

We showed a decrease in the VL fascicle angle during eccentric contraction (from $17.1^{\circ}$ at short muscle length angles to $12.2^{\circ}$ at long muscle length angles in the IT eccentric mode vs. $18.2^{\circ}$ to $11.9^{\circ}$ in the IK mode). Previous studies have obtained similar results in isometric (Fukunaga et al. 1997) or in eccentric contractions (from $18^{\circ}$ to $14^{\circ}$ ) (Reeves and Narici 2003). The mechanical load differences - notably, the differences in external torque and angular velocity - did not induce mode-specific (IT or IK) modifications of the fascicle angle as a function of the joint angle. These results are in accordance with the study by Reeves and Narici (2003), which did not reveal any effect of angular velocity on the fascicle angle of TA in eccentric contractions.

IT and IK eccentric exercises load the muscle-tendon system differently based on the angular velocity and torque. Previous works on dynamic conditions showed no effect of movement velocity on muscle architecture parameters during eccentric contractions (Chino et al. 2009; Reeves and Narici 2003). However, it seems that exercise intensity has an impact on muscle fascicle behavior (Pasquet et al. 2006). Thus, the instantaneous torque differences observed between the IT and IK eccentric modes suggest specific changes of muscle architecture in each mode. However, comparative analysis of IT and IK standardized eccentric exercises did not reveal such differences, which invalidates our hypothesis. The torque differences observed in our study can be partly explained by the differences in the neural drive of VL observed between the two modes. Despite these differences in EMG activity, the IT and IK modes induce similar behavior in VL architecture, which may not exclude different behavior of three others heads of quadriceps femoris. Previous studies have shown that maximal contractions are associated with a greater EMG activity at a short muscle length than at long muscle length of tibialis anterior (Pasquet et al. 2005) or vastus lateralis (Babault et al. 2003), which is consistent with the higher EMG activity concomitant to higher torque levels in IT mode at short muscle length. Otherwise, it has already been shown that passive torque significantly increases with stretching velocity, especially at high torque levels, illustrating the viscoelastic behavior of the muscle-tendon complex (Nordez et al. 2008). Thus, the higher instantaneous velocity in the IT mode at a short muscle length could influence the viscoelastic structures of the muscle-tendon unit, which could also participate in generating the higher external torques produced in this condition. Muscle thickness slightly decreased from short to long muscle length during eccentric contractions, perhaps because of the muscle lengthening and the decrease of fascicle angle observed during eccentric contraction, regardless of the exercise mode in eccentric contraction. According to Maxwell et al.'s (1974) model, such changes in fascicle angle and fascicle length result in decreased muscle thickness.

In conclusion, the mechanical differences between IT and IK eccentric exercises (i.e., external torque, angular velocity) did not result in specific behavior of the muscle architecture. Nevertheless, this study is the first to determine the changes in muscle thickness, fascicle angle and fascicle length of vastus lateralis during both IT and IK eccentric contractions in vivo. Results showed that VL fascicles lengthen during eccentric contraction, as previously reported in a high-intensity stretch-shortening cycle (Ishikawa et al. 2003). This increase in muscle fascicle length suggests that muscle-tendon lengthening during eccentric contraction is supported by both muscle fibers and the patellar tendon during IT and IK eccentric contractions. Fascicle angle simultaneously decreases from short to long muscle length, as shown in other contexts, in the contracting muscle (Fukunaga et al. 1997; Reeves and Narici 2003). Those variations result in a decrease of muscle thickness through the eccentric action. The differences in external torque and angular velocity between the IT and IK modes are concomitant to specific neural patterns of the agonist and antagonist EMG activity. Such differences suggest that specific adaptations of the neural drive may occur after IT or IK eccentric training, as shown by previous studies performed on concentric training (Remaud et al. 2010). Future research is needed to provide new insights into this issue.

Acknowledgments This study was supported by grants from the «Région des Pays de la Loire» and the «Réseau Recherche et Sport en Pays de la Loire» (France).

Conflict of interest Authors had no conflict of interest. 
References

Aagaard P, Simonsen EB, Trolle M, Bangsbo J, Klausen K (1995) Isokinetic hamstring/quadriceps strength ratio: influence from joint angular velocity, gravity correction and contraction mode. Acta Physiol Scand 154:421-427

Abellaneda S, Guissard N, Duchateau J (2009) The relative lengthening of the myotendinous structures in the medial gastrocnemius during passive stretching differs among individuals. J Appl Physiol 106:169-177

Babault N, Pousson M, Michaut A, Van Hoecke J (2003) Effect of quadriceps femoris muscle length on neural activation during isometric and concentric contractions. J Appl Physiol 94:983990

Bigland B, Lippold OC (1954) The relation between force, velocity and integrated electrical activity in human muscles. J Physiol 123:214-224

Bland JM, Altman DG (1986) Statistical methods for assessing agreement between two methods of clinical measurement. Lancet 1:307-310

Blazevich AJ, Gill ND, Zhou S (2006) Intra- and intermuscular variation in human quadriceps femoris architecture assessed in vivo. J Anat 209:289-310

Burden A, Bartlett R (1999) Normalisation of EMG amplitude: an evaluation and comparison of old and new methods. Med Eng Phys 21:247-257

Chino K, Mitsukawa N, Kobayashi K, Miyoshi Y, Oda T, Kanehisa H, Fukunaga T, Fukashiro S, Kawakami Y (2009) The influence of fascicle behavior on the lack of velocity dependence in eccentric joint torque in humans: in vivo observation. J Appl Biomech 25:111-118

Duchateau J, Enoka RM (2008) Neural control of shortening and lengthening contractions: influence of task constraints. J Physiol 586:5853-5864

Duclay J, Martin A (2005) Evoked H-reflex and V-wave responses during maximal isometric, concentric, and eccentric muscle contraction. J Neurophysiol 94:3555-3562

Duclay J, Martin A, Robbe A, Pousson M (2008) Spinal reflex plasticity during maximal dynamic contractions after eccentric training. Med Sci Sports Exerc 40:722-734

Enoka RM (1996) Eccentric contractions require unique activation strategies by the nervous system. J Appl Physiol 81:2339-2346

Fukunaga T, Ichinose Y, Ito M, Kawakami Y, Fukashiro S (1997) Determination of fascicle length and pennation in a contracting human muscle in vivo. J Appl Physiol 82:354-358

Fukunaga T, Kubo K, Kawakami Y, Fukashiro S, Kanehisa H, Maganaris CN (2001) In vivo behaviour of human muscle tendon during walking. Proc Biol Sci 268:229-233

Guilhem G, Cornu C, Guével A (2010a) Neuromuscular and muscletendon system adaptations to isotonic and isokinetic eccentric exercise. Ann Phys Rehabil Med 53:319-341

Guilhem G, Cornu C, Nordez A, Guevel A (2010b) A new device to study isoload eccentric exercise. J Strength Cond Res 24: 3476-3483

Guilhem G, Guével A, Cornu C (2010c) A standardization method to compare isotonic vs. isokinetic eccentric exercises. J Electromyogr Kinesiol 20:1000-1006

Herbert RD, Gandevia SC (1995) Changes in pennation with joint angle and muscle torque: in vivo measurements in human brachialis muscle. J Physiol 484(Pt 2):523-532

Hermens HJ, Freriks B, Disselhorst-Klug C, Rau G (2000) Development of recommendations for SEMG sensors and sensor placement procedures. J Electromyogr Kinesiol 10:361-374

Hopkins WG (2000) Measures of reliability in sports medicine and science. Sports Med 30:1-15
Housh DJ, Housh TJ, Weir JP, Weir LL, Evetovich TK, Donlin PE (1998) Effects of unilateral eccentric-only dynamic constant external resistance training on quadriceps femoris cross-sectional area. J Strength Cond Res 12:192-198

Ichinose Y, Kawakami Y, Ito M, Kanehisa H, Fukunaga T (2000) In vivo estimation of contraction velocity of human vastus lateralis muscle during "isokinetic" action. J Appl Physiol 88:851-856

Ishikawa M, Finni T, Komi PV (2003) Behaviour of vastus lateralis muscle-tendon during high intensity SSC exercises in vivo. Acta Physiol Scand 178:205-213

Junior VA, Bottaro M, Pereira MC, Andrade MM, Júnior PPR, Carmo JC (2010) Electromyographic analyses of muscle pre-activation induced by single joint exercise. Rev Bras Fisioter 14:158-165

Kellis E, Baltzopoulos V (1998) Muscle activation differences between eccentric and concentric isokinetic exercise. Med Sci Sports Exerc 30:1616-1623

Komi PV, Linnamo V, Silventoinen P, Sillanpaa M (2000) Force and EMG power spectrum during eccentric and concentric actions. Med Sci Sports Exerc 32:1757-1762

Kubo K, Kanehisa H, Azuma K, Ishizu M, Kuno SY, Okada M, Fukunaga T (2003) Muscle architectural characteristics in young and elderly men and women. Int J Sports Med 24:125-130

Maxwell LC, Faulkner JA, Hyatt GJ (1974) Estimation of number of fibers in guinea pig skeletal muscles. J Appl Physiol 37:259-264

Mullany H, O'Malley M, St Clair Gibson A, Vaughan C (2002) Agonist-antagonist common drive during fatiguing knee extension efforts using surface electromyography. J Electromyogr Kinesiol 12:375-384

Nakazawa K, Kawakami Y, Fukunaga T, Yano H, Miyashita M (1993) Differences in activation patterns in elbow flexor muscles during isometric, concentric and eccentric contractions. Eur J Appl Physiol Occup Physiol 66:214-220

Nardone A, Romano C, Schieppati M (1989) Selective recruitment of high-threshold human motor units during voluntary isotonic lengthening of active muscles. J Physiol 409:451-471

Nordez A, Casari P, Cornu C (2008) Effects of stretching velocity on passive resistance developed by the knee musculo-articular complex: contributions of frictional and viscoelastic behaviours. Eur J Appl Physiol 103:243-250

Pasquet B, Carpentier A, Duchateau J (2005) Change in muscle fascicle length influences the recruitment and discharge rate of motor units during isometric contractions. J Neurophysiol 94:3126-3133

Pasquet B, Carpentier A, Duchateau J (2006) Specific modulation of motor unit discharge for a similar change in fascicle length during shortening and lengthening contractions in humans. J Physiol 577:753-765

Pinniger GJ, Steele JR, Thorstensson A, Cresswell AG (2000) Tension regulation during lengthening and shortening actions of the human soleus muscle. Eur J Appl Physiol 81:375-383

Reeves ND, Narici MV (2003) Behavior of human muscle fascicles during shortening and lengthening contractions in vivo. J Appl Physiol 95:1090-1096

Remaud A, Cornu C, Guével A (2009) Agonist muscle activity and antagonist muscle co-activity levels during standardized isotonic and isokinetic knee extensions. J Electromyogr Kinesiol 19:449-458

Remaud A, Cornu C, Guével A (2010) Neuromuscular adaptations to 8-week strength training: isotonic versus isokinetic mode. Eur J Appl Physiol 108:59-69

Sleivert GG, Wenger HA (1994) Reliability of measuring isometric and isokinetic peak torque, rate of torque development, integrated electromyography, and tibial nerve conduction velocity. Arch Phys Med Rehabil 75:1315-1321

Visnes H, Bahr R (2007) The evolution of eccentric training as treatment for patellar tendinopathy (jumper's knee): a critical review of exercise programmes. Br J Sports Med 41:217-223 